\title{
La frontera-gulag y las deportaciones de migrantes mexicanos
}

\author{
GUILLERMO ALONSO MENESES
}

El control cada vez más estricto y agresivo de Estados Unidos en su frontera con México presenta en 1993 y 2001 dos momentos clave que explican su actual statu quo. En los próximos años seguirá encontrando "razones" en la amenaza del terrorismo yihadista, el tráfico de drogas y el tránsito indocumentado de migrantes para reforzar la vigilancia y construir una frontera con más tramos "impermeables". Esta realidad fronteriza ha hecho más vulnerables a las y los migrantes: del lado sur se encuentran a merced del crimen organizado, y del lado norte, de la infraestructura, el modus operandi de los vigilantes y de las políticas, legislación y persecución interna que se traducen en deportaciones duras de afrontar. Las detenciones, las deportaciones y los miles de muertos de sólo los últimos siete años evidencian cambios importantes en el proceso-flujo migratorio.

PALABRAS CLAVE: migrantes, vulnerabilidad, frontera, deportaciones, Estados Unidos

\section{The Gulag-border and Mexican Migrant Deportations}

The increasing strict and aggressive border control that the United States has in its border with Mexico had two key moments in 1993 and 2001 that can explain the current statu quo. In the coming years the threat of jihadist terrorism, drug trafficking, and undocumented migrants crossing are enough "reasons" to strength surveillance and build more "sealed" points. This border has actually turn the migrants more vulnerable to potential damage: at the south side they are between of organized crime and in the north side infrastructure, modus operandi of the border patrol, policies, legislation and internal persecution result in deportations that are hard to face. Apprehensions, removed events and deaths counted in just the past seven years show that there have been major changes in the flow-process of migrants crossing.

El Colegio de la Frontera Norte, 
n un ensayo sobre las fronteras, el escritor Salman Rushdie (2003) comenta una imagen de la serie Exodus del fotógrafo brasileño Sebastião Salgado, tomada frente a Tijuana, a la altura de la colonia Libertad —el escritor indobritánico la ubica en un lugar impreciso de la frontera México-Estados Unidos-, en la que se ve en la distancia la silueta de un migrante que corre desesperado de regreso hacia la barda de metal, hacia México, mientras un vehículo todo terreno de la Patrulla Fronteriza se dirige a él a toda velocidad por un camino de tierra. La imagen en blanco y negro es de gran dramatismo y refleja claramente lo absurdo de estas escenas en un contexto fronterizo como el del suroeste de Estados Unidos (EUA). Rushdie definió aquella "pared entre Estados Unidos y México"1 de finales del siglo pasado — Salgado tomó las fotos de Exodus entre 1993 y 1999 - como una mezcla “entre la Gran Muralla china y el gulag" (Rushdie, 2003: 354). ${ }^{2}$ Tras vincularlo con otros casos, Rushdie habló de "fronteras de un mundo-gulag" (Rushdie, 2003: 356), ${ }^{3}$ o lo que es peor, del mundo occidental concebido como un gulag.

Antes de continuar, debo explicar dos claves de lectura. 1) Hablo de fronteragulag porque hay tramos frente a Tijuana, que no son los únicos, en los que la infraestructura fronteriza evoca esas imágenes de campos de concentración nazis o de gulags soviéticos: alambradas, altas bardas y portalones de acero, cámaras, vigilantes, perros, iluminación nocturna, patrullas y otros artefactos intimidatorios. Aquel muro que Sebastião Salgado fotografió y Salman Rushdie describió como propio de un gulag era un juguete herrumbroso inservible comparado con lo que hay hoy en día. 2) En este trabajo hago una lectura antropológica — desde la experiencia y la sensibilidad aportada por el trabajo de campo y la interacción con los actores- de la vulnerabilidad subyacente a la experiencia de los migrantes que primero cruzaron de manera clandestina y después de vivir años como inmigrantes con el temor a ser descubiertos terminaron deportados. Esto a partir de un conjunto de discursos oficiales, mediáticos y académicos que se expresan en términos estadísticos, legislativos, periodísticos y teóricos. No es que desconfie del discurso social y de las

\footnotetext{
1 "Wall between United States and Mexico" [traducciones libres del autor, n. del ed.].

2 "Part Great Wall of China, part gulag".

3 "Border gulag-world".
} 
declaraciones de gente real que hace cosas reales, los protagonistas de la experiencia migratoria, pero en esta ocasión decidí privilegiar esta información.

\section{Algunos antecedentes}

La frontera que separa EuA de México continúa fortificándose y se muestra como fachada y eje de un espacio hipervigilado e inexpugnable. Este despliegue de poder envía un doble mensaje: a los migrantes irregulares para que se lo piensen dos veces y a los electores estadounidenses para mostrar que no se ha perdido el control sobre la frontera, máxime ahora que el proyecto de ley que se impulsa para regularizar al colectivo de más de 11 millones de migrantes indocumentados en EUA, la Border Security, Economic Opportunity, and Immigration Modernization Act, plantea que debe reforzarse la seguridad de la frontera antes de cualquier regularización.

Sin embargo, la demanda de una "frontera segura" degeneró en frontera-gulag, inspirada en una moral política que tiene mucho de la orwelliana novela 1984 y del Big Brother, que todo lo vigila. Esta nueva categoría de control fronterizo combina los muros y los altos cercos de acero enrejados con espacios y carreteras intermedios, además de un entramado de cámaras de distinto espectro y sensores regulados por software y computadoras, que elevan la dificultad de cruzar sin ser detectado, a lo que se suman desde 2004 los drones o aviones teledirigidos para vigilancia. Dicha exhibición de fuerza se localiza sobre todo junto a las ciudades fronterizas mexicanas, pues aún quedan tramos en montañas y desiertos donde las construcciones responden a una tipología menor de muros y obstáculos concebidos para impedir el paso de vehículos y no de personas, e incluso donde la infraestructura es mínima o inexistente.

Una inversión tan descomunal en infraestructura, en teoría para impedir todo cruce, algo tiene de campo de concentración del siglo XXI, pero semejante heteroestructura tecnológica no parece estar ahí para detener sólo el flujo de migrantes que entran a EUA sin documentos migratorios. Los argumentos esgrimidos, o si se prefiere la coartada para levantar una frontera-prisión de alta seguridad, fueron en primer lugar la prevención de las amenazas del terrorismo islamista-yihadista inspirado en Al-Qaeda después de 2001; en segundo lugar atajar los cruces relacionados con el crimen organizado y el narcotráfico que se han intensificado en los últimos 20 años, y en tercer lugar el paso de la corriente migratoria irregular. Obviamente, el presente es producto de una serie de políticas y medidas implementadas en respuesta a complejas circunstancias históricas a uno y otro lado de la frontera. Encontramos algunos de los antecedentes hacia el final del Programa Bracero en 1964, la crisis del petróleo de 1973-1975 y los sexenios de José López Portillo y Miguel de la Madrid (1976-1988) hasta la actualidad, caracterizada por la Gran Recesión sobrevenida en 2008 y el saldo migratorio neto cercano a cero en México en 2011 (Passel, Cohn y González-Barrera, 2012). Un lapso de 50 años que otros autores, como Jorge Durand, dividen para efectos de manipulación analítica en periodos de aproximadamente 20 años.

Durand (2012), en una síntesis que encapsula prácticamente un siglo en una columna de opinión, nos recuerda que la etapa que denomina "indocumentada” (1965-1986) culminó “con una ley de amnistía y un programa especial para trabajadores agrícolas (IRCA, por sus siglas en inglés)" "4 que legalizó a 2.3 millones de mexicanos. La siguiente etapa, que denomina "bipolar" (1986-2007), terminó antes de la crisis, cuando se calculaba que en EuA había 12 millones de migrantes nacidos en México, de los que 6500 son indocumentados. La última etapa 


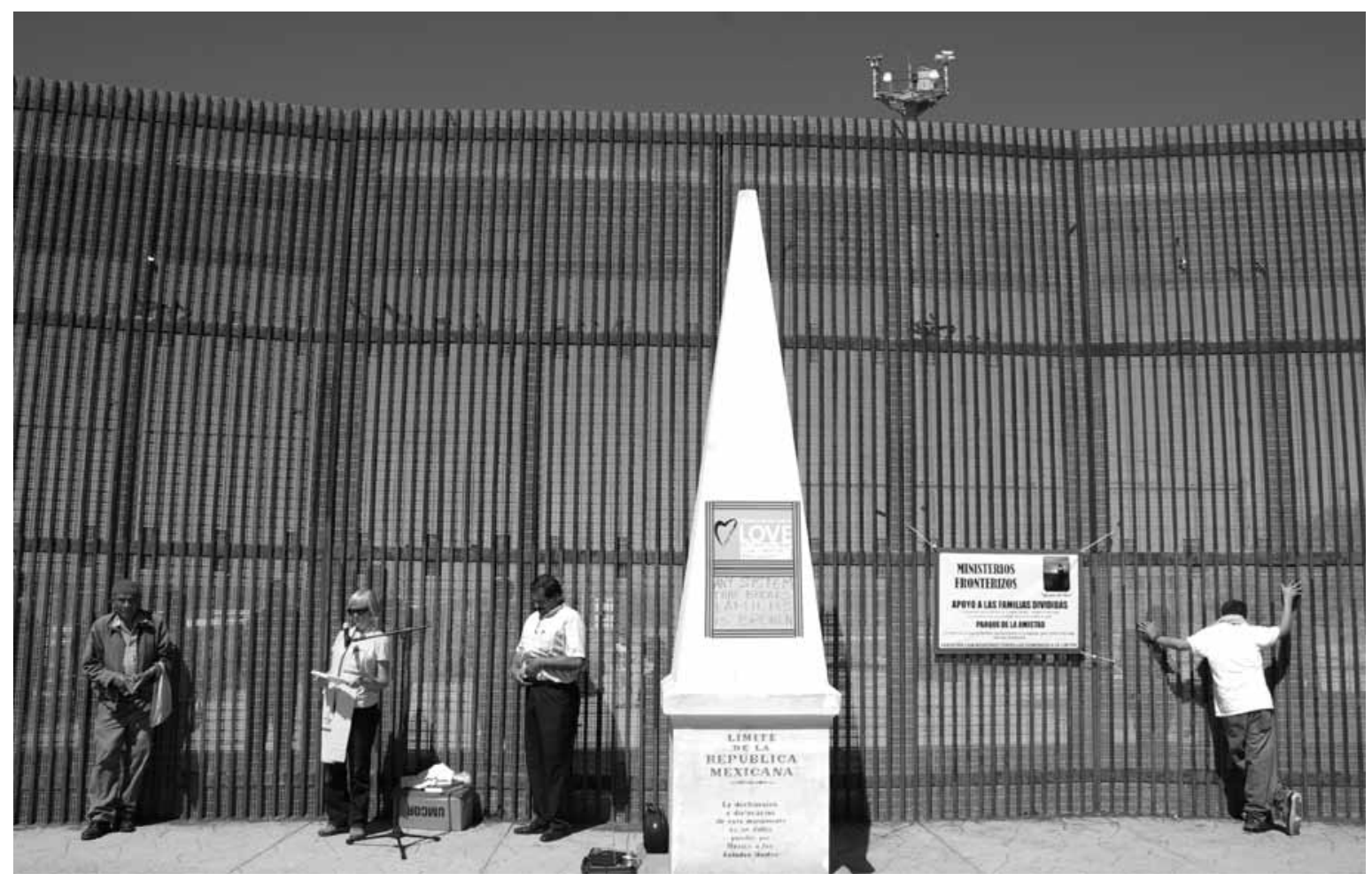

Guillermo Alonso Meneses D Doble barda desde Playas de Tijuana durante un oficio religioso, invierno de 2014.

continuaría abierta —Durand dixit- y habría iniciado en 2008. Desde entonces el fenómeno entró en un proceso de reversión: "las entradas subrepticias, que se siguen dando, se compensan con las deportaciones".

Aquí interesa una etapa diferente, definida cronológicamente por el periodo 1993-2013, durante la cual EUA endureció en sucesivas etapas el cruce de indocumentados sur-norte y el combate de la amenaza terrorista, el trasiego de drogas y el contrabando — smuggling - y tráfico — trafficking o la antigua trata- de migrantes. "Contrabando" y "tráfico" es la terminología vigente a partir de los Protocolos de Palermo de diciembre de 2000, suscritos por la mayoría de Estados en la Convención de las Naciones Unidas contra la Delincuencia Organizada Transnacional. Sólo que el contrabando de armas, municiones, dólares y el pequeño flujo de migrantes indocumentados estadounidenses que fluye de norte a sur ha sido poco perseguido. Si acaso, el United States Customs and Border Protection (USCBP) levanta registros de tanto en tanto a la entrada de México, por ejemplo, por San Ysidro, California, pero el caos vial que provoca lo hace inviable de manera permanente.

El resultado ha sido la construcción de una frontera-gulag en tramos estratégicos y una política de deportación deshumanizada y socialmente destructiva, que empujó al flujo de migrantes y a las comunidades de inmigrantes irregulares a una situación crónica y estructural de indefensión y vulnerabilidad ante toda clase de peligros y humillaciones, altas tasas de robo con violencia, asesinatos y muertes acaecidas por la dureza del cruce, la explotación laboral, la violación sistemática de los derechos de menores de edad y la separación de familias, entre otras consecuencias. 


\section{La estigmatización de la migración irregular y la fortificación de la frontera}

Los actuales muros y la política de deportaciones son inseparables de la predominante ideología antiinmigrante de amplias capas sociales y de políticos. Nevins, al referirse a los inmigrantes indocumentados —illegal aliens — en EUA, habló de "las raíces ideológicas de lo ilegal" (Nevins, 2002: 95 y ss.). ${ }^{5}$ Obviamente, existe una retroalimentación entre esta ideología y la concepción del control policiaco-militar de una frontera, pero ni los muros ni la ideología bastan para detener un flujo migratorio consolidado. Es necesario implementar controles internos al interior de los países (Doomernik, 2010: 28). Éstos van más allá de los checkpoints, retenes o redadas, porque responden a acciones concebidas y articuladas para garantizar el funcionamiento del aparato del Estado, un entramado de postulados ideológicos interiorizados por los distintos gobernantes y gobiernos. Kessler se refiere a esta dimensión a partir del enfoque de la "gubernamentalidad" propuesto por Foucault, que surge de interrelacionar factores como la seguridad, el territorio y la población por parte de un gobierno para controlar e incidir en lo que Kessler interpreta como la "conducta de las conductas" foucaultiana (Kessler, 2009: 34). De este modo las fronteras más difíciles de franquear o más agresivas con las personas, la naturaleza y las comunidades reflejan la mentalidad, la ideología, los fundamentos morales de los estilos de gobierno que están detrás de esta "gubernamentalidad". También Sandra Gil analizó el entramado de prácticas de control fronterizo contemporáneas en el caso europeo desde las perspectivas de las tecnologías de gobierno y la proliferación de instrumentos que obstaculizan la movilidad de "los otros", con base en el enfoque de los "dispositivos" foucaultianos para otorgarle centralidad analítica a las mentalidades y modalidades de gobierno (Gil, 2011: 22-24). El resultado apunta a que las fronteras fortificadas son una solución acorde a estilos o modalidades de gobierno fruto de partidos políticos con ideologías agresivas hacia el extranjero, con arraigo social, sin necesidad de que exista un peligro objetivo.

Sin pretender ni poder ser exhaustivos, para el caso de la frontera México-Estados Unidos varios autores han aportado nuevas perspectivas de análisis que dan razón de los principales factores, cambiantes con los años, tanto gubernamentales (Dunn, 1996; Andreas, 2000; Cornelius y Tsuda; 2004), sustanciados en lo que el geógrafo Nevins denominó "el paisaje del control y el miedo" (Nevins, 2002: 144), que ya caracterizaba a la frontera a fines de la década de 1990, como de los relacionados con los migrantes, como la tesis de la "resistencia hormiga" de los migrantes en un contexto de "apartheid global" (Spener, 2009). Sea como fuere, el principal problema es la inmensidad del espacio fronterizo y un "enemigo" prometeico que - paradójicamente - puede ser un terrorista yihadista, un narcotraficante violento o un migrante desorientado.

La frontera México-Estados Unidos - estrictamente la terrestre/fluvial tiene una extensión aproximada de 3146 kilómetros - durante décadas tuvo un statu quo laxo del que se beneficiaron de forma disímil los migrantes y la sociedad/economía estadounidense. Sin embargo, en la década de 1970 el sistema-mundo capitalista (Amin et al., 1999) comenzó a ensayar nuevas modalidades de explotación o negocios para aprovechar la coyuntura internacional de crisis económica aguda, incluida la estigmatización de los trabajadores y los colectivos de inmigrantes. Este periodo de 40 años comprendido entre la crisis del petróleo de 1973-1975, incluida la Gran Recesión (Krugman dixit, 2009) declarada en el otoño de 2008, hasta el presente, establecen el marco histórico de la situación "reciente" en la frontera caracterizado por la vulnerabilidad de los (in)migrantes.

\footnotetext{
5 "The ideological roots of ilegal".
} 
Hacia 1975, el panorama internacional estaba definido por la Guerra Fría entre el occidente capitalista y el bloque comunista. Estados Unidos “dejó" la Guerra de Vietnam en 1973 y el 6 de octubre del mismo año estalló la Guerra del Yom Kipur entre árabes e israelíes. Semanas después se produjo una reducción por boicot en la producción de hidrocarburos y una escalada de precios que desembocó en la crisis del petróleo de 1973-1974. Cuando las economías occidentales se resintieron, ya estaban dadas las condiciones propicias para que la hoguera xenófoba y antiinmigrante prendiera. Hubo un deterioro de la percepción pública de los inmigrantes irregulares residentes en EUA, el chivo expiatorio clásico cuando hay crisis en la economía, y en esta "agresión" jugaron un papel importante los medios de comunicación que distorsionaron la agenda política migratoria de aquellos años. Una situación que ilustraron tempranamente Bustamante (1979) y Cornelius (1979). El primero nos recordó que en EuA la prensa de la época hablaba de los inmigrantes indocumentados en términos de "una invasión de ilegales", "invasión silenciosa", un caso de "crisis nacional" (The New York Times, 1974, citado en Bustamante, 1979), una "carga de 13000 millones de dólares para los contribuyentes" (Bustamante, 1979: 23). Wayne Cornelius sintetizaba así lo ocurrido:

La administración de Carter ha hecho más que cualquiera de las precedentes para elevar el nivel de preocupación entre la población estadounidense en general, sobre la inmigración indocumentada. [...] Ya para el año 1976, aproximadamente un 80 por ciento o más del pueblo estadounidense creía que los migrantes indocumentados acaparan los trabajos de los habitantes legales, que se meten en actividades criminales, que rebajan el nivel de los salarios que se pagan a los habitantes legales, y que muchos recogen pagos por desempleo o "welfare" (Cornelius, 1979: 28-30).
Resulta significativo que aquellos estereotipos antiinmigrantes sigan vigentes, que el expresidente Jimmy Carter recibiera el Nobel de la Paz en 2002 y que el Carter Center luche por los derechos humanos, la democracia y el bienestar de la población mundial. Las tempranas críticas de Cornelius conectan con la tesis de Dunn (1996) cuando señala que la militarización de la frontera y la adopción de la doctrina del conflicto de baja intensidad se consolidaron durante 1978-1992, es decir, inició bajo el mandato Carter, concluyó "formalmente" con Bush padre y conllevó un aumento del maltrato de migrantes y la violación de sus derechos humanos.

Esta percepción negativa de la migración o animadversión contra lo que por entonces se denominaba "inmigrantes procedentes del Tercer Mundo" también se manifestó en Europa, sólo que en forma de "nuevas retóricas de exclusión" (Stolcke, 1995). Y es que "el rechazo a los migrantes que se encuentran en forma no autorizada residiendo en determinadas sociedades no sólo responde a crisis económicas sino también a actitudes xenofóbicas" (Verea, 2012b: 42). De hecho, trabajos recientes detectan un esquema similar de la ideología antiinmigrante vigente aún, como los de Gilbert y Kolnick (2012), Solop y Wonders (2012) o Joysmith (2012).

La sociedad estadounidense arrastró durante todo el siglo xx una cultura racista que permeó a lo largo y ancho de la nación. La segregación racial, un régimen de apartheid que operaba de facto, no “terminó" oficialmente hasta la promulgación de la Civil Rights Act de 1964, el mismo año en que acabó el Programa Bracero, pero también cuando el antropólogo William Madsen (1964) publicaba que los migrantes eran asaltados de manera sistemática y denunciaba la existencia de auténticas "cacerías" cuando eran baleados desde la orilla estadounidense en el área de McAllen, en el condado de Hidalgo. Respecto de las circunstancias de cruce clandestino escribió: "Algunos de los espaldas mojadas jamás llegaron a cruzar. Se ahogaron 


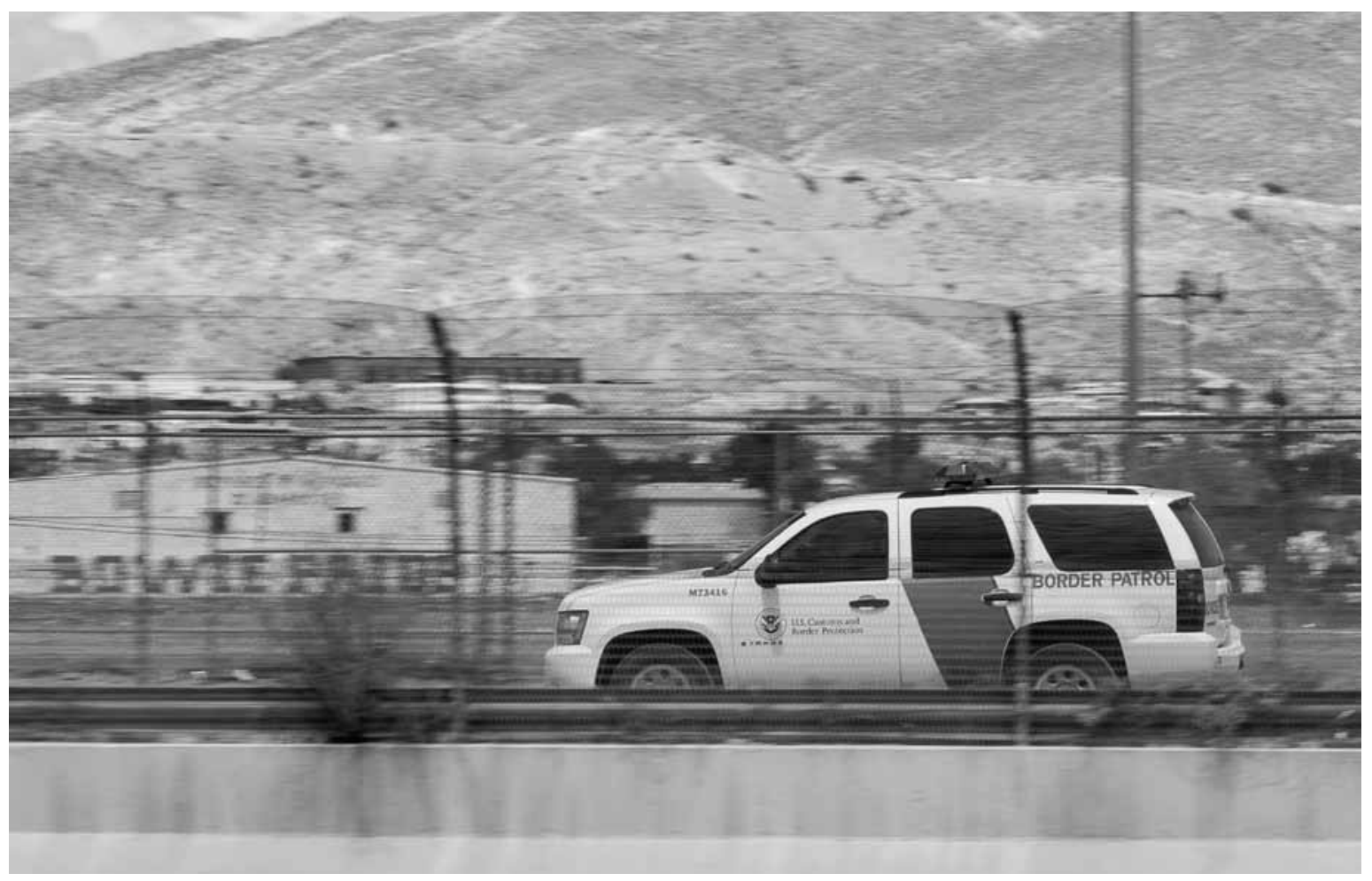

Prometeo Lucero - Frontera Ciudad Juárez-El Paso.

o les dispararon misteriosamente desde la orilla de Estados Unidos y sus cuerpos quedaron varados en los bancos de arena del río" (Madsen, 1964: 25). ${ }^{6}$ Décadas después, Mike Davis habla de las fotos de mexicanos asesinados a tiros por la espalda que siendo niño llegó a ver en Ocotillo Wells, Imperial, en el sur de California, aproximadamente en la década de 1950: "Les dispararon a todos [jóvenes mexicanos, espaldas mojadas]. Por la espalda"" (Davis, 2002: ix).

Este tipo de violencia estructural e histórica es inseparable de la ideología racista y criminal hegemónica interiorizada en la cultura de buena parte de los estadounidenses "caucásicos", por utilizar la terminología confusa o delirante del U. S. Census Bureau, sobre todo en la frontera con México (McWilliams, 1968) y que inevitablemente afloró también entre los agentes de la Patrulla Fronteriza (Hernández, 2004; 2010) y todavía hoy se muestra bajo distintas formas y esferas públicas (Verea, 2012a). Está enquistada en la "gubernamentalidad" o, si se prefiere, en "las mentalidades y modalidades de gobierno" en EUA.

Siguiendo la tesis de Dunn, tampoco fue casual que coincidieran en el tiempo el recrudecimiento del conflicto de baja intensidad en la frontera y el clima de animadversión contra los migrantes. Los actuales operativos en la frontera con México responden a un largo periodo de acciones planificadas del desaparecido Immigration and Naturalization Services (INS) y otras agencias o el ejército — por

6

"Some of the wetbacks never made the crossing. They were drowned or mysteriously shot from the American shore and their bodies were washed up on the river banks". "They [young Mexican men, wetbacks] were all shot. In the back". 
ejemplo, la Joint Task Force 6 (JTF-6)-, a raíz de la guerra contra las drogas — war on drugs- que data del mandato de Nixon y que con Reagan se relanzó, al incorporar este objetivo al trabajo del INS y la Border Patrol de la época (Dunn, 1996: 80; 2001: 8-9; Meyers, 2005: 4).

\section{Los operativos de la Border Patrol y la trama legislativa 1993-2013}

Bill Clinton llegó a la presidencia de EuA en enero de 1993 y sorpresivamente el INs puso en marcha el 19 de septiembre la "Operation Blockade" —Operación Bloqueo- en el tramo del sector de El Paso, ubicado entre Sunland Park, Nuevo México - conurbado con El Paso-, y Fabens, en Texas. Semanas después, tras las protestas de México, el INs lo renombró con el políticamente correcto "Hold the Line" (Echbach et al., 1999: 448) y reformuló su estrategia. Fue la primera medida antiinmigrante en la frontera de la administración demócrata en un contexto de crisis económica heredada de las deficitarias administraciones republicanas de Reagan y Bush padre. Si el nombre y el operativo "Blockade" fueron un ensayo de semanas, su metamorfosis en "Hold the Line" inició una época de hierro y sin retorno en la lucha contra la migración irregular. El detonante fue la conjunción de factores económicos depresivos, la ideología antiinmigrante y la asunción demócrata de una "gubernamentalidad" heredada frente a la migración clandestina y el control fronterizo, sobre todo cuando las señales provenientes desde México comenzaron a aparecer en enero de 1994 con el levantamiento del Ejército Zapatista de Liberación Nacional (EZLN).

La administración Clinton, presionada por amplios sectores antiinmigrantes de la sociedad, las prioridades de la "agenda oculta" de agencias como el INS, la Drug Enforcement Administration (DEA) o el Pentágono, inició una nueva época de control fronterizo. Las inercias históricas del flujo migratorio procedente del sur pronto se fracturaron. A las iniciativas "Blockade/Hold the Line" les siguieron las operaciones "Gatekeeper" en octubre de 1994 en San Diego, California, y "Safeguard” en Nogales, Arizona, a principios de 1995, aunque esta última no se puso en operación en todo el sector de Tucson y posteriormente en Yuma sino hasta 1999. La "Operation Rio Grande" en Texas se instrumentó en agosto de 1997 y se extendió por el bajo Río Grande entre Brownsville y Laredo.

Posteriormente ha habido operativos menores o especiales como complemento de los principales. Por ejemplo, a mediados de 2004 se implementó el operativo "Arizona Border Control" o ABC, en el que se utilizaron por primera vez de manera oficial los drones o unmanned aerial vehicles (UAV) para vigilancia, así como un programa de repatriación voluntaria que incluía el pago del viaje al centro de México.

El impacto de estos operativos se reflejó pronto en las cifras oficiales de detenciones. Con "Hold the Line" el sector de El Paso pasó de realizar 285781 aprehensiones en el año fiscal (AF) de 1993 a 79688 en 1994 - la “Operación Bloqueo” inició 11 días antes de que acabara ese año fiscal- Las fechas de las estadísticas oficiales de EUA se refieren a años fiscales, que desde 1976 van del 1 de octubre al 30 de septiembre del año siguiente. Así, el AF 1994 inició el 1 de octubre de 1993. Estas cifras se refieren a eventos de detenciones, no de personas, por lo que alguien pudo ser recapturado y contabilizado varias veces.

Aquel descenso de las detenciones provocó un impacto de distinto sentido en uno y otro lado de la frontera. El Paso había sido durante décadas el segundo sector con mayor número de detenciones en la frontera con México, superado sólo por el excepcional sector de San Diego, pero desde 1994 fue desplazado por los sectores de McAllen ahora rebautizado Rio Grande Valley y Tucson. Este desplazamiento geográfico de las rutas migratorias implicó 
CUADRO 1. DETENCIONES POR SECTORES Y AÑO FISCAL (1993-1996, 2006-2008, 2011-2013)

\begin{tabular}{|c|c|c|c|c|c|c|c|c|c|c|}
\hline Año & 1993 & 1994 & 1995 & 1996 & 2006 & 2007 & 2008 & 2011 & 2012 & 2013 \\
\hline El Paso & 285781 & 79688 & 110971 & 145929 & 122256 & 75464 & 30312 & 10345 & 9678 & 11154 \\
\hline $\begin{array}{l}\text { San } \\
\text { Diego }\end{array}$ & 531689 & 450152 & 524231 & 483815 & 142104 & 152460 & 162390 & 42447 & 28461 & 27496 \\
\hline Tucson & 92639 & 139473 & 227529 & 305348 & 392074 & 378239 & 317696 & 123285 & 121000 & 120939 \\
\hline
\end{tabular}

Fuente: Elaborado con datos del usDHS.

nuevos lugares y estrategias de cruce. La actual insignificancia estadística del sector de El Paso en lo que respecta a intentos de cruce y detenciones debe explicarse asimismo por la crisis económica y las secuelas de la violencia en la vecina Ciudad Juárez.

El sector de San Diego, colindante con Tijuana y entrada natural a California, también sufrió cambios radicales. Desde 1973 y durante 24 años fue el sector con el mayor número de detenciones de migrantes anuales en la frontera. El récord se estableció el año del IRCA en 1986, con 629656 detenciones -39\% del total-, mientras que para el conjunto de sectores fronterizos se hicieron 1615844 - hablamos siempre de eventos de detenciones-. De este modo, si en 1993 hubo 531689 detenciones, 1997 fue el último año en que este sector registró el mayor número de eventos: 283889. Ya en 1998 Tucson ocupó el primer lugar. De manera inesperada, en 2008 hubo un repunte en San Diego que lo colocó en el segundo lugar nacional tras Tucson, cayó cuando parecía resucitar en 2011 y de nuevo en 2012 y 2013 fue superado por los sectores de Rio Grande Valley y Tucson.

El sector de Tucson, Arizona, se mantuvo 14 años con el número más elevado en reportes de detenciones. El extenso territorio desértico con sierras escarpadas que va de Nuevo México a Yuma, colindante con California, alejado de las ciudades fronterizas importantes y con carreteras que conectan con los mercados laborales de California, Nevada, la misma Arizona y Texas, está detrás de su "éxito". Al inicio de los operativos en 1993 era el cuarto sector en detenciones, en 1994 el segundo y en 1998 con 387406 aprehensiones ocupó el primer lugar hasta 2012. Fue desplazado por el sector de Rio Grande Valley — antes McAllen — en 2013. El récord de detenciones en Tucson data del año 2000 con 616346 de un total de 1649884 en la frontera con México. Las cifras del cuadro 1 y su desplazamiento espacial (de)muestran que el grueso del flujo de migrantes se movió a los desiertos con su climatología implacable y letal, en parajes desolados donde la pronta ayuda es imposible.

Un evento capital de este periodo, una legislatura demócrata, fue la promulgación de la Illegal Immigration Reform and Immigrant Responsibility Act (IIRIRA o IIRAIRA) en 1996, una ley que conllevó el endurecimiento en la detención y deportación de indocumentados. La IIRIRA favoreció el levantamiento de "barreras y cercas físicas", ${ }^{8}$ la contratación de más agentes para la Patrulla Fronteriza, autorizó la transferencia y el uso de cualquier tecnología o equipamiento en poder del Estado a toda agencia gubernamental o la aplicación de tecnologías para la identificación y registro de datos como los del programa IDENT o el United States Visitor and Immigrant Status Indicator Technology (Usvisit) (Meyers, 2005: 10-13). Una década después, en 2006, se impulsó una serie de acuerdos y

8 
programas amparados por la Immigration and Nationality Act Section 287(g) vinculada a la IIRIRA.

Los siguientes datos nos dan una idea del impacto real de los operativos en aquellos años sobre la inmigración. Entre octubre de 1994 y septiembre de 2000 se acumularon 8844476 eventos de detenciones en la frontera con México. Paradójicamente, la población mexicana en EUA se duplicó entre 1980-1990 y de nuevo entre 1990-2000 (Passel, Cohn y González-Barrera, 2012: 21). Es decir, casi 9 millones de detenciones en seis años no sólo no evitaron que la población mexicana indocumentada se duplicara en una década, sino que estimularon su tránsito ilegal por la frontera: cuando el cruce se puso difícil, las estancias se alargaron durante años. $\mathrm{Al}$ mismo tiempo se promulgaron leyes que repercutieron en la protección de migrantes y en la tipificación de delitos vinculados a la migración, como la Victims of Trafficking and Violence Protection Act del 28 de octubre del 2000 para combatir el tráfico o trata de personas y la violencia contra las mujeres y menores, reforzada después con la Trafficking Victims Protection Reauthorization Act de 2003. Estas leyes fueron el reconocimiento oficial de que al interior del proceso social migratorio hay facetas criminales y confirmó la voluntad de combatirlas.

La Patrulla Fronteriza batió el récord anual de detenciones de migrantes en su frontera con México en el AF 2000, aunque ya desde junio de ese año el número de detenciones comenzó a descender de manera acentuada respecto del mismo mes del año anterior, más de 14 meses antes de los fatídicos atentados de 2001. Es decir, el flujo migratorio como se refleja con las detenciones en la frontera con México empezó a caer desde junio de 2000, cuando se contaron 115000 aprehensiones —en junio de 1999 se habían hecho aproximadamente 119000-, y no desde septiembre de 2001. Al finalizar el AF 2001 el 30 de septiembre se confirmó el descenso en las detenciones durante más de 14 meses seguidos, al contabilizar 1235718 frente al 1643679 de 2000 .
El dato de la caída del flujo migratorio reflejado en detenciones fue eclipsado por los atentados del 11 de septiembre de 2001, que forzaron una nueva era en la seguridad e impactaron en el estatus de la frontera y en el proceso migratorio. El 26 de octubre el presidente firmó la Patriot Act, cuyo nombre oficial es Uniting and Strengthening America by Providing Appropriate Tools Required to Intercept and Obstruct Terrorism Act, que sentó las bases jurídicas para crear el Departamento del Homeland Security (USDHS). Al año siguiente, el 14 mayo de 2002 se promulgó la Enhanced Border Security and Visa Entry Reform Act (EBSVERA) y el 25 de noviembre la Homeland Security Act, que suele ser traducida como "Seguridad de la Patria o Nacional", aunque la ley establece: "Cada uno de los términos 'la patria americana' o 'la patria' se refieren a los Estados Unidos de América". ${ }^{9}$ Esta nutrida legislación sentó las bases de una reorganización de distintas oficinas y agencias que derivó en la abolición del viejo INS en marzo de 2003 y la aparición de nuevas corporaciones, como el Immigration and Customs Enforcement (ICE), el United States Citizenship and Immigration Services (USCIS) o el Customs and Border Protection (USCBP), entre otras, así como en la inyección de miles de millones de dólares del presupuesto a seguridad, lo que se reflejó en rubros como la infraestructura fronteriza y el de patrulleros de frontera.

Las cifras del cuadro 2 contabilizan el total de agentes de la Patrulla Fronteriza para toda la nación, que no se ha elevado desde 2011, sin embargo cerca de $90 \%$ están adscritos a sectores de la frontera con México. Este incremento implicó desde 1993 un importante aumento de las horas de vigilancia en la línea (Escobar, Bean y Weintraub, 1999), pie en tierra, y por tanto en la capacidad de detención de

9 "Each of the terms American homeland and homeland means the United States". 
CUADRO 2. NÚMERO DE AGENTES DE LA PATRULLA FRONTERIZA EN TODA LA NACIÓN

\begin{tabular}{cccccccc} 
Año & $\mathbf{1 9 9 2}$ & $\mathbf{1 9 9 5}$ & $\mathbf{2 0 0 0}$ & $\mathbf{2 0 0 7}$ & $\mathbf{2 0 0 8}$ & $\mathbf{2 0 0 9}$ & $\mathbf{2 0 1 1}$ \\
\hline Patrulleros & 4139 & 4945 & 9212 & 14923 & 17499 & 20119 & 21444 \\
\hline
\end{tabular}

Fuente: Elaborado con datos del Ins y del usDHS.

inmigrantes ilegales. La canalización de mayores recursos a estas actividades continuó gracias a los imperativos de la Intelligence Reform and Terrorism Prevention Act del 2004, la Emergency Supplemental Appropriations Act for Defense, the Global War on Terror, and Tsunami Relief Act (REAL ID) del 4 de enero de 2005 o la Secure Fence Act del 26 de octubre de 2006.

Estas leyes sirvieron para implementar medidas que hoy son realidad o que deterioraron las libertades de nacionales y extranjeros y aseguraron el incremento de efectivos y el uso de tecnologías avanzadas (Meyers, 2005: 18; uscis, 2006). Por ejemplo, la Secure Fence Act de 2006 establece en su Sección 2 (a) (1) y (2) que el control operacional de la frontera implica la vigilancia de las fronteras marítimas y terrestres con el uso de personal y tecnología como “aviones teledirigidos, sensores de detección sobre el terreno, satélites, radares y cámaras de vigilancia", ${ }^{10}$ el desarrollo de infraestructuras físicas o como "puestos de control adicionales, carreteras a prueba de inclemencias meteorológicas y barreras contra vehículos". ${ }^{11}$ Por último, la Sección 2 (b) define el término "control operacional" —operational controlcomo "la prevención de todas las entradas a los Estados Unidos que no estén apegadas a la ley, incluida la entrada de terroristas, otros extranjeros irregulares, instrumentos del terrorismo, narcóticos u otro tipo de contrabando" (Uscis, 2006). ${ }^{12}$ Así se dotó de contenido al concepto de "frontera-gulag" de un Estado que estaba en guerra contra el terrorismo. Paralelamente, el clima ideológico y político que propició estas leyes está detrás de legislaciones locales como la SB 1070 en Arizona para controlar la migración indocumentada con criterios ilegales, involucrando a cuerpos de policías locales o al sheriff Arpaio, lo que unido a la capacidad de aprehensión, al registro electrónico de la información biométrica de los detenidos y al aumento de camas en centros de detención ha operado como un factor eficaz que acaba cansando, intimidando y disuadiendo a los migrantes.

\section{El descenso de los cruces indocumentados y el aumento de las deportaciones}

El siglo Xxi había comenzado con la crisis de los rancheros caza inmigrantes en el sur de Arizona y desde junio de 2000 — aunque en marzo de aquel año se estableció el récord de mayor número de detenciones para un solo mes, con 220000 - el flujo anual de las detenciones comenzó a caer. Hubo un ligero repunte en 2004 y 2005 con 1139282 y 1171396 detenciones, respectivamente, que coincidió con los Minutemen antiinmigrantes y el resurgimiento de la añeja inercia xenófoba. En medio de aquellas tensiones, en agosto de 2005 los gobernadores de Nuevo México y Arizona decretaron el estado de emergencia por la violencia del narcotráfico en la frontera. La caída en la cifra de detenciones fue drástica en el periodo 2005-2011.

\footnotetext{
10 "Unmanned aerial vehicles, ground-based sensors, satellites, radar coverage, and cameras".

11 "Additional checkpoints, all weather access roads, and vehicle barriers".

12 "The prevention of all unlawful entries into the United States, including entries by terrorists, other unlawful aliens, instruments of terrorism, narcotics, and other contraband".
} 


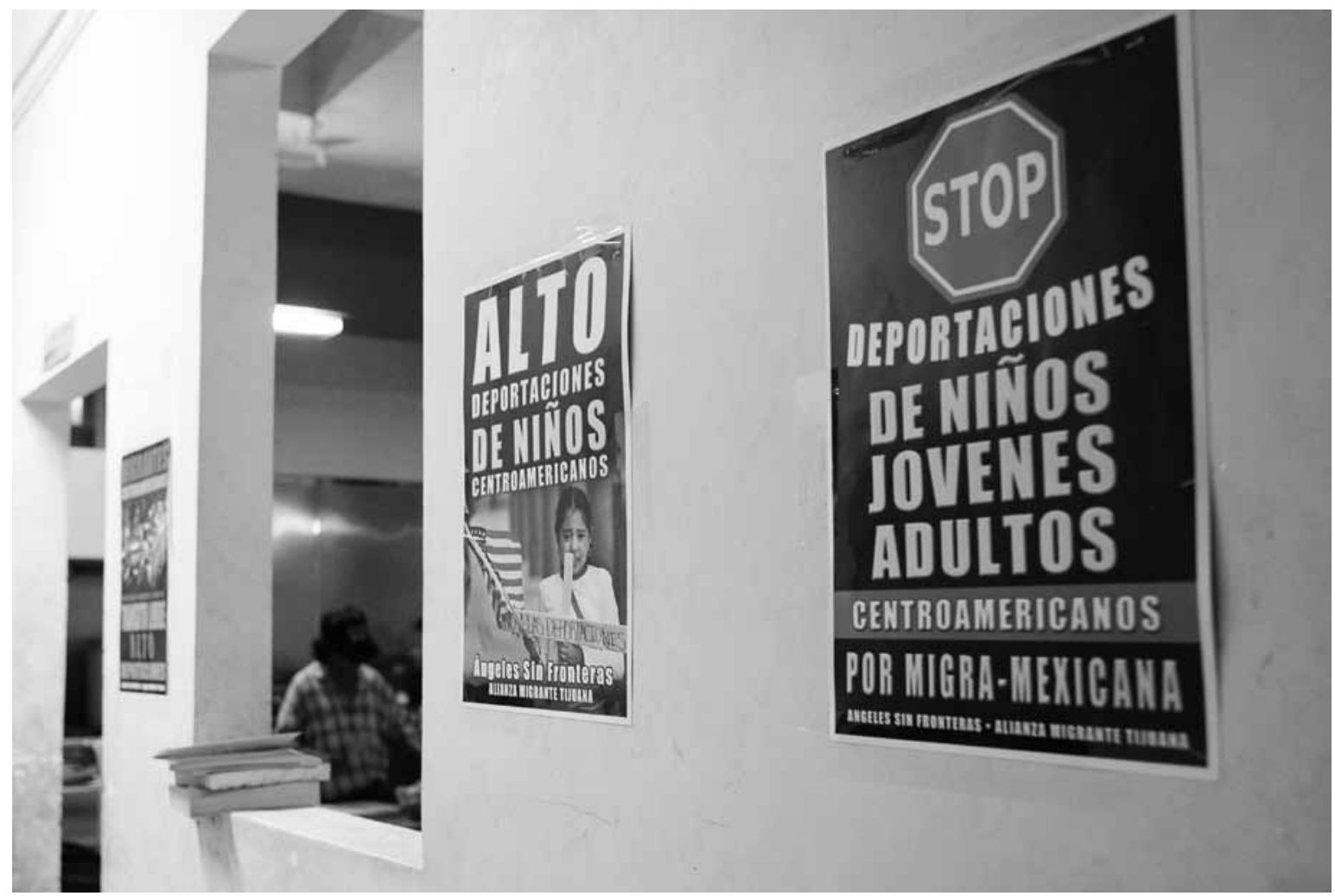

Prometeo Lucero Hotel Migrante, Mexicali.

Pero en la frontera-gulag no todo son detenciones de migrantes irregulares. Para hacernos una idea de la intensidad de interacciones que enfrenta EUA en sus fronteras a diario, sirva de muestra esta información: en 2012, en promedio diario, se admitieron 963121 pasajeros y visitantes que cruzaron por tierra; 66615 contenedores transportados por tráiler — truck - tren o barco; se realizaron 999 detenciones en las garitas de entrada o en campo abierto; se arrestó a 54 criminales con una orden de búsqueda y se rechazó a 931 extranjeros inadmisibles en los puertos de entrada; se requisaron 11660 libras de droga —aproximadamente 5288 kilogramos-y 274065 dólares en dinero no declarado (USCBP, 2013c). La dimensión de filtro en los puertos y puertas de entrada resulta obvia, pero el número de interacciones es difícil de controlar de manera exhaustiva.
Sin embargo, aunque el cruce de la frontera disminuyó, los residentes indocumentados en EUA aumentaron hasta 2007 debido a un aspecto que ya se apuntó desde finales de la década de 1990: el descenso significativo de los retornos (Tuirán, 2000): "En realidad, el efecto más contundente de todas estas medidas [militarización de la frontera, incremento de la capacidad de detener, etc.] ha sido reducir la tasa de retorno de los migrantes indocumentados" (Massey, Pren y Durand, 2009: 119).

Si bien los retornos voluntarios disminuyeron o se dilataron en el tiempo y coincidieron con una recuperación económica vinculada a la industria de la guerra y a la burbuja inmobiliaria, a partir de 2007 comenzaron a crecer notablemente las deportaciones o remociones - removals - con orden judicial. Los confusos registros del ICE prácticamente se 
duplicaron en el bienio 2008-2009 con lo peor de la crisis. Es importante desagregar el dato de las deportaciones totales y tener claro el concepto, porque la administración de Obama no es la que ha realizado el mayor número de deportaciones - el récord para un año lo tienen Reagan en 1986 y Clinton en 2000_, sino la que ha llevado a cabo el mayor número de deportaciones o removals en la modalidad de "delincuente, inmigrantes fugitivos y deportados del interior" - “criminal offender, immigration fugitives e interior removal"-. El desconcierto y la confusión en los últimos años por las estadísticas de deportaciones es problemático (Alonso, 2012).

Paralelamente a las detenciones -apprehensions - y expulsiones — returned - de la Border Patrol-CBP, el ICE, primero bajo el mandato de Bush y después de Obama, comenzó a aplicar intensamente la política de incrementar las redadas, las investigaciones y las deportaciones en sus distintas modalidades en ciudades y localidades alejadas de la frontera. El ICE empezó a funcionar en 2003 y ha operado cambios en las actividades y formas de contabilización. Si en 2004 se registraron 202842 deportaciones o remociones - la mayoría de mexicanos-, desde 2007 se superan las 300000 anuales - mayores y menores de edad de ambos sexos, la mayoría mexicanos-.

El ICE desagrega la cifra de removals en varias categorías, por ejemplo, en 2013 deportó — removed a 368644, de los cuales 133551 fueron detenidos al interior del país. De éstos, 82\% había sido condenado por un crimen, mientras que del número total de las deportaciones 59\% o 216810 habían sido condenados - convicted - previamente por un crimen o delito. Así, "98\% del total de deportaciones de la agencia [ICE] fueron criminales convictos, migrantes recién cruzados, migrantes sin permiso, reincidentes o aquellos que ya habían sido deportados por el ICE, todos estos casos están alineados con las prioridades a combatir por la agencia" (ICE, 2014a). ${ }^{13}$

Asimismo, de las 235093 deportaciones o remociones que realizó el ICE de individuos detenidos en la frontera en 2013, casi 95\% fueron capturados por la Patrulla Fronteriza en campo abierto mientras intentaban entrar irregularmente - unlawfully- y luego fueron entregados al ICE, que los fichó — processed-y deportó. El 5\% restante fue retenido en los puertos de entrada por oficiales del USCBP. El origen de los 368644 deportados fue: México, 241 493; Guatemala, 47 769; Honduras, 37 049; El Salvador, 21 602, y República Dominicana, 2 462, principalmente (ICE, 2014a).

Es decir, detrás de la categoría "deportadoremoved" hallamos casos tan distintos como un migrante que recién cruzó la frontera, un criminal convicto o un residente irregular durante más de 20 años. Por este motivo no es cierto que la administración de Obama haya deportado a más inmigrantes que Bush o Clinton. La confusión surge porque a partir de 2004, pero en especial después de 2009, se ha cambiado la naturaleza de los componentes del registro de removals o de lo que hemos traducido como "deportaciones".

Las detenciones reportadas en los sectores de la frontera con México han ido en descenso. En el periodo 2007-2013 hubo 3651089 (USCBP, 2013a), la mayoría de mexicanos y no todos optaron por salir/ retornar "voluntariamente", pues varios millares impugnan cada año ante un juez su expulsión, o bien son identificados como criminales y puestos a disposición judicial. Las cifras reflejan una caída tan radical e inédita del flujo migratorio que las detenciones de 2011 recuerdan las 321326 aprehensiones de 1972. Hallamos así que mientras las detenciones caían, las deportaciones crecían: en el periodo 20072013 se realizaron 2636983 eventos de deportación - removals - en tanto que durante el lapso 2001-2006 fueron 1333362 (USDHS, 2011: 4). La

"98 percent of the agency's total removals were convicted criminals, recent border crossers, illegal re-entrants or those previously removed by ICE, in line with agency's enforcement priorities". 


\begin{tabular}{ccccccccc}
\multicolumn{6}{l}{ CUADRO 3. EVENTOS DE DEPORTACIONES Y DE DETENCIONES EN LA FRONTERA 2006-2013 } \\
\multicolumn{1}{c}{ Año } & $\mathbf{2 0 0 6}$ & $\mathbf{2 0 0 7}$ & $\mathbf{2 0 0 8}$ & $\mathbf{2 0 0 9}$ & $\mathbf{2 0 1 0}$ & $\mathbf{2 0 1 1}$ & $\mathbf{2 0 1 2}$ & $\mathbf{2 0 1 3}$ \\
\hline Deportaciones & 280974 & 319382 & 359795 & 395165 & 387242 & 396906 & 409849 & 368644 \\
\hline Detenciones & 1071972 & 858638 & 705022 & 540851 & 447731 & 327577 & 356873 & 414397 \\
\hline
\end{tabular}

* De las deportaciones, los AF 2006-2010 (USDHS, 2011), de loS AF 2011 y 2012 (ICE, 2013), AF 2013 (ICE, 2014a).

Fuente: USDHS.

diferencia entre detenciones y deportaciones para el periodo 2007-2013 arroja un saldo a favor de las detenciones de 1014106 eventos. Al menos de esta cifra no se infiere un aumento espectacular de las deportaciones del interior. Éstas sólo superaron a las detenciones en la frontera en el bienio 2011-2012.

Es decir, en el periodo 2001-2013 las deportaciones ascendieron a 3970345 eventos, la mayoría con destino México. Sin embargo, estas cifras oficiales deberán revisarse, desagregarse y refinarse categorialmente ante la acusación de que hubo presión para imponer cuotas de detenciones al ICE (Hsu y Becker, 2010), que fueron infladas para "calmar" a los republicanos (González, 2012) y porque hubo y hay una confusión de conceptos y componentes — no son coherentes - al integrar la cifra de deportados-removed (Alonso, 2012). Esto se desprende también de recientes informes del ICE (2014a y 2014b).

Por otro lado, las deportaciones tienen una dimensión de limpieza étnica y de cirugía de extirpación socioeconómica. De facto, son una forma de deshacerse de individuos estigmatizados o incómodos, illegal aliens, y no sólo porque algunos son reclusos que suponen un gasto. Esto conlleva efectos colaterales, otra faceta de la vulnerabilidad de los inmigrantes indocumentados, ya que existe un flujo de retornos formalmente "voluntarios" entre la población indocumentada, intimidada por las redadas del ICE o de otras corporaciones acogidas a convenios amparados por la Section 287 (g), misma que se une a la corriente indirecta de "expulsados", que formalmente son "retornados voluntarios" a México porque no hubo intermediación del ICE, miles de ellos con la ciudadanía estadounidense. Passel, Cohn y González-Barrera incluyen entre ellos a los hijos de migrantes nacidos en EUA:

De 2005 a 2010, 1.4 millones de mexicanos y sus familias (incluyendo menores con ciudadanía estadounidense por nacimiento) dejaron Estados Unidos para trasladarse a México, según cifras del Censo mexicano de 2010. Esto supone aproximadamente el doble de los 670000 que lo hicieron una década antes, de 1995 a 2000. Mientras que la mayoría de estos inmigrantes regresaron voluntariamente, un estimado entre 5\% y 35\% de ellos retornaron como resultado de las deportaciones habidas entre 2005 y 2010 (Passel, Cohn y González-Barrera, 2012: 11). ${ }^{14}$

Finalmente, en el lado mexicano de la frontera ha habido una presencia activa y agresiva del crimen organizado, que ha demostrado sobradamente ser cruel y sanguinaria con los migrantes, aparte de que ha puesto al descubierto un entramado secundario de delincuentes y funcionarios que buscan lucrar. A

14

"From 2005 to 2010, 1.4 million Mexicans and their families (including U.S.-born children) left the U.S. to move to Mexico, according to data from the 2010 Mexican census. That is about double the 670000 who did so a decade earlier, from 1995 to 2000 . While most of these immigrants returned voluntarily, an estimated 5\% to 35\% returned as a result of deportations between 2005 and 2010". 
partir del periodo 1998-2002, que coincide con la irrupción de los Zetas, se registró un mayor número de robos, violaciones, extorsiones, secuestros y asesinatos de migrantes. En parte porque la frontera-gulag los "embalsa" temporalmente en la región y los expone más tiempo a la violencia del crimen organizado, con el agravante de que estas organizaciones cobran derecho de piso a los "coyotes" y los migrantes llegan a operar como moneda de cambio.

El resultado fue y es un aumento de la vulnerabilidad de los migrantes mexicanos, así como de los centro y sudamericanos, víctimas recurrentes de extorsión, persecución violenta o secuestro para el negocio criminal (Bustamante, 2011; Durand, 2011), actividades denominadas por Hernández de León (2012) como la "industria bastarda de la migración". Este problema se redimensionó con la aparición a fines de agosto de 2010 de los cuerpos masacrados de 58 hombres y 14 mujeres en un rancho de San Fernando, Tamaulipas, todos migrantes en tránsito, la mayoría de México, y otros hondureños, salvadoreños, ecuatorianos y brasileños. La situación se completa con un movimiento de desplazamientos y exilio en México (Aguilar, 2012; Notimex, 2012).

Así, el descenso de la migración coincidió con un repunte de las deportaciones y con el periodo más sangriento de la denominada "guerra contra el narco" de 2006 a 2012, que incluye el trienio sangriento de 2010 a 2012 en el que se superaron las 14000 muertes violentas anuales (Escalante, 2011; Silva, Pérez y Gutiérrez, 2012), pero también con un repunte de la violencia de la Patrulla Fronteriza:

De acuerdo con una investigación de 2013 del diario The Arizona Republic, agentes estadounidenses de la Patrulla Fronteriza han asesinado a 42 personas desde 2005. Algunos de ellos, como el adolescente José Antonio Elena Rodríguez, fueron asesinados del lado mexicano de la frontera por disparos de agentes estadounidenses realizados a través del muro (Di Cintio, 2014). ${ }^{15}$
Ahora bien, si el escenario más drástico y mortal para los migrantes está en la región fronteriza, si hubo más de 30 millones de eventos de cruces, detenciones y deportaciones durante el periodo 1993-2013 y la mayor parte se originó previamente en un cruce clandestino por lugares peligrosos, esto en conjunto explica las aproximadamente 8600 víctimas mortales en el viaje de internamiento ilegal en Estados Unidos. Las causas de muerte son bien conocidas, pero sobresalen cinco factores letales básicos: 1) insolación-hipertermia, 2) ahogamiento, 3) hipotermia, 4) atropellos y accidentes vehiculares y 5) asesinato con arma de fuego. Éstas no son las únicas, pero sí las más importantes por su incidencia, las encontramos a lo largo de toda la frontera y se repiten año tras año (Echbach et al., 1999; Alonso, 2013).

\section{(In)conclusiones}

La migración México-EUA experimentó profundos cambios en los últimos 50 años, desde el final del Programa Bracero en 1964, pero en particular durante el periodo 1993-2013. En concreto, aunque en los periodos 1993-2000 y 2001-2006 se sentaron fuertes bases legislativas, económicas o de doctrina de seguridad para marcar una ruptura de las tendencias históricas, fue en el excepcional y complejo periodo 2007-2013 que la migración México-EUA desembocó en un escenario que no había sido previsto: saldo migratorio cercano a cero, descenso de los cruces irregulares y las detenciones cerca de la frontera, aumento de las deportaciones de migrantes de largo arraigo.

15

"According to a 2013 investigation by The Arizona Republic newspaper, American border patrol agents have killed 42 people since 2005. Some, like the teenager José Antonio Elena Rodríguez, were killed on the south side of the border by American agents shooting through the wall into Mexico". 
Desde el 1 de octubre de 1993 a la actualidad se acumularon más de 20 millones de eventos de detención de migrantes en la frontera del suroeste estadounidense con México. El colapso sufrido por los flujos migratorios se explica por una concurrencia de factores económico-laborales, la fronteragulag, el apoyo de una legislación migratoria que se contaminó con el problema de la seguridad nacional o el combate al narcotráfico, en el que la frontera es clave, y aseguró la inversión de miles de millones de dólares, pero con un impacto negativo en los derechos humanos y civiles.

Otro factor fueron las redadas del ICE y la política de deportaciones del USDHS con distintas modalidades, que si en 2004 hicieron 202842 deportaciones - la mayoría de mexicanos - pasaron a promediar desde 2007 más de 350000 deportados anuales - mayormente mexicanos-, con 409000 en el 2012, el récord con la nueva forma de contabilidad del ICE. Ahora bien, estas deportaciones sólo superaron a las detenciones en la frontera en el bienio 20112012, lo que nos indica que las cifras oficiales deberán revisarse, desagregarse y refinarse categorialmente.

Asimismo, las deportaciones tienen un trasfondo de limpieza-étnica y de cirugía de extirpación socioeconómica cada vez más nítido, porque afectan sobre todo a inmigrantes irregulares de México, Centroamérica y el Caribe: hispanoparlantes de piel morena. Esto ha conllevado el retorno forzado de familias con ciudadanos estadounidenses en calidad de cónyuges, parejas o hijos a México. Y por falta de previsión, ciertos deportados han causado problemas y conflictos locales en algunas ciudades fronterizas como Tijuana, algo previsible en toda población flotante inesperada.

Los daños colaterales causados por la política estadounidense de construir fronteras-gulag e intensificar las deportaciones, sumados a la creciente vulnerabilidad de los migrantes ante distintas formas de violencia, son una poderosa razón para repensar las relaciones México-Estados Unidos. Esto incluiría algunos debates polémicos: la libre circulación de trabajadores o la doctrina de "fronteras abiertas", la legalización de las drogas, la prohibición de la venta de armas de alto poder, la transformación de la economía capitalista y la erradicación de la pobreza y la desigualdad, entre otros rubros. Pero esto ya es materia de otro artículo. D

\section{Bibliografía}

Aguilar, Julián, 2012, "Forced North by Drug Wars, but United in Exile", en The New York Times, 13 de abril, p. $21 \mathrm{~A}$.

Alonso Meneses, Guillermo, 2012, "Recesión económica, reflujos migratorios y violencia antiinmigrante entre México y Estados Unidos”, en Norteamérica. Revista Académica del CISAN-UNAM, año 7, núm. 2.

—_, 2013, El desierto de los sueños rotos. Detenciones y muertes de migrantes en la frontera México-Estados Unidos, 1993-2013, El Colegio de la Frontera Norte, México.

Amin, Samir et al., 1999, Dinámica de la crisis global, Siglo XXI, México.

Andreas, Peter, 2000, Border Games Policing the U. S.-Mexico Divide, Cornell University Press, Ithaca.

Anguiano, María Eugenia y Ana María López (eds.), 2010, Migraciones y fronteras. Nuevos contornos para la movilidad internacional, Icaria, Centre d'Informació i Documentació Internacionals a Barcelona, Barcelona.

Baumann, Mechthild, Astrid Lorenz y Kerstin Rosenow (eds.), 2011, Crossing and Controlling Borders. Immigration Policies and their Impact on Migrants' Journey, Budrich Unipress (Political Sciences), Opladen y Farmington Hills.

Bustamante, Jorge, 1979, "Inmigración indocumentada a los Estados Unidos", en El Colegio de México, Indocumentados, mitos y realidades, El Colegio de México, México, pp. 23-60.

__. 2011, "Secuestro de migrantes", en Reforma, 9 de noviembre, México. 
Cintio, Marcelo di, 2014, "The Walls that Hurt Us", en The New York Times, 23 de enero, en línea: <http://www.nytimes.com/2014/01/24/opinion/the-walls-that-hurt-us.html?src=rechp\&_r=1>, consultado el 30 de enero de 2014.

Colegio de México, El, 1979, Indocumentados, mitos y realidades, El Colegio de México, México.

Cornelius, Wayne A., 1979, "La migración ilegal mexicana a los Estados Unidos: conclusiones de investigaciones recientes, implicaciones políticas y prioridades de investigación", en El Colegio de México, Indocumentados, mitos y realidades, El Colegio de México, México.

__ Philip L. Martin y James F. Hollifield (eds.), 2004, Controlling Immigration: A Global Perspective, Stanford University Press, Stanford.

__ y Takeyuki Tsuda, 2004, “Controlling Immigration: The Limits of Government Intervention”, en Wayne A. Cornelius, Philip L. Martin y James F. Hollifield (eds.), Controlling Immigration: A Global Perspective, Stanford University Press, Stanford, pp. 3-48.

Davis, Mike, 2002, "Foreword", en Joseph Nevins, Operation Gatekeeper. The Rise of the "Illegal Alien" and the Making of the U S.-Mexico Boundary, Routledge, Nueva York.

Doomernik, Jeroen, 2010, "Del permiso a la prisión: una exploración multidisciplinar de las interacciones entre procesos migratorios e intervención estatal”, en María Eugenia Anguiano y Ana María López (eds.), Migraciones y fronteras. Nuevos contornos para la movilidad internacional, Icaria, Centre d'Informació i Documentació Internacionals a Barcelona, Barcelona.

Dunn, Timothy J., 1996, The Militarization of the U. S.-Mexico Border, 1978-1992. Low-Intensity Conflict Doctrine Comes Home, Center for Mexican American Studies-University of Texas, Austin.

__ 2001, "Border Militarization Via Drug and Immigration Enforcement: Human Rights Implications”, en Social Justice, vol. 28, núm. 2, pp. 7-30.

Durand, Jorge, 2011, "El secuestro de migrantes", en La Jornada, 20 de noviembre, en línea: <http://www.jornada.unam.mx/2011/11/20/ politica/018a2pol>

—_, 2012, "Nueva etapa migratoria”, en La Jornada, 12 de agosto, en línea: <http://www.jornada.unam.mx/2012/08/12/politica/022a2pol>. Echbach, Karl et al., 1999, "Death at the Border”, en International Migration Review, vol. 33, núm. 2, pp. 430-454.

Escalante Gonzalbo, Fernando, 2011, “Homicidios 2008-2009. La muerte tiene permiso”, en Nexos, núm. 397, en línea: <http://www.nexos. com.mx/?p=14089>, consultado el 30 de enero de 2014.

Escobar, Agustín, Frank D. Bean y Sidney Weintraub, 1999, La dinámica de la emigración mexicana, Centro de Investigaciones y Estudios Superiores en Antropología Social, Miguel Ángel Porrúa, México.

Gil, Sandra, 2011, "Reinventing Europe's Borders: Delocalization and Externalization of Eu Migration Control through the Involvement of Third Countries", en Mechthild Baumann, Astrid Lorenz y Kerstin Rosenow (eds.), Crossing and Controlling Borders. Immigration Policies and their Impact on Migrants' Journey, Budrich Unipress (Political Sciences), Opladen y Farmington Hills.

Gilbert, Liette y Kathy A. Kolnick, 2012, "Constitutional Failure or Anti-Immigrant Success? Local Anti-Immigrant Ordinances and Sentiments in the United States", en Mónica Verea (ed.), Sentimientos, acciones y politicas antiinmigrantes. América del Norte y la Unión Europea, Centro de Investigaciones sobre América del Norte-Universidad Nacional Autónoma de México, México, pp. 83-102.

González, Ricard, 2012, “Eeuv 'engordó' la cifra de deportaciones para paliar las críticas republicanas”, en El Mundo, en línea: <www.elmundo. es/america/2010/12/06/estados_unidos/1291655313.html>.

Hernández, Kelly Lytle, 2004, "Ni blancos ni negros: mexicanos. El papel de la Patrulla Fronteriza estadounidense en la definición de una nueva categoría racial. 1924-1940", en Cuicuilco, vol. 11, núm. 31, pp. 1-20.

, 2010, Migra!: A History of the U. S. Border Patrol, University of California Press, Berkeley.

Hernández de León, Rubén, 2012, “La industria de la migración”, conferencia, Seminario Permanente de Migración, 9 de marzo, El Colegio de la Frontera Norte, Tijuana.

Hsu, Spencer S. y Andrew Becker, 2010, "ICE Officials Set Quotas to Deport More Illegal Immigrants”, en The Washington Post, 27 de marzo.

Immigration and Customs Enforcement (ICE), 2013, "FY 2013 ICE Immigration Removals", en línea: <http://www.ice.gov/removal-statistics/>, consultado el 17 de julio de 2013.

_- 2014a, "FY 2013: ICE Announces Year-end Removal Numbers", en línea: <http://www.ice.gov/news/releases/1312/131219washingtondc. htm>, consultado el 30 de enero de 2014

__, 2014b, “Fy 2013 ICE Immigration Removals. ERo Annual Report”, en línea: <https://www.ice.gov/doclib/about/offices/ero/pdf/2013-iceimmigration-removals.pdf>, consultado el 30 de enero de 2014.

Joysmith, Claire, 2012, "'Godzilla con sombrero de charro': visiones testimoniales y el imaginario anti(in)migrante estadounidense post-11 de septiembre", en Mónica Verea (ed.), Sentimientos, acciones y políticas antiinmigrantes. América del Norte y la Unión Europea, Centro de Investigaciones sobre América del Norte-Universidad Nacional Autónoma de México, México, pp. 191-206. 
Kessler, Gabriel, 2009, El sentimiento de inseguridad, sociología del temor al delito, Siglo XXI, Buenos Aires.

Krugman, Paul, 2009, "How Did Economists Get it so Wrong?", en The New York Times, 2 de septiembre.

Madsen, William, 1964, Mexican-Americans of South Texas, Holt, Rinehart and Winston, Nueva York.

Massey, Douglas, Karen Pren y Jorge Durand, 2009, "Nuevos escenarios de la migración México-Estados Unidos. Las consecuencias de la guerra antiinmigrante", en Papeles de Población, vol. 15, núm. 61, pp. 101-128.

McWilliams, Carey, 1968, Al norte de México: conflicto entre "anglos" e "hispanos", Siglo XXI, México.

Meyers, Deborah W., 2005, “U. S. Border Enforcement: From Horseback to High-Tech”, en Insight, núm. 7, en línea: <http://www.migrationpolicy.org/research/us-border-enforcement-horseback-high-tech>.

Moreno, Carolina, 2012, "Border Crossing Deaths more Comon as Illegal Immigration Declines”, en Huffington Post, Sección Latino Voices, 17 de octubre, Nueva York, en línea:<http://www.huffingtonpost.com/2012/08/17/border-crossing-deaths-illegal-immigration_n_1783912. html>, consultado el 13 de febrero de 2013.

Nevins, Joseph, 2002, Operation Gatekeeper. The Rise of the "Illegal Alien" and the Making of the U. S.-Mexico Boundary, Routledge, Nueva York. Notimex, 2012, "Narco desplaza a 160000 mexicanos: ACnuR", en El Economista, 19 de abril, México.

Passel, Jeffrey y D’Vera Cohn, 2011, “Unauthorized Immigrant Population: National and State Trends, 2010”, Pew Research Center, Washington, D. C., en línea: <www.pewhispanic.org/2011/02/01/unauthorized-immigrant-population-brnational-and-state-trends-2010/>, consultado el 16 de marzo de 2012.

__ y Ana González-Barrera, 2012, "Net Migration from Mexico Falls to Zero -and Perhaps Less", Pew Research Center, Washington, D. C., en línea: <http://www.pewhispanic.org/2012/04/23/net-migration-from-mexico-falls-to-zero-and-perhaps-less/>.

Rushdie, Salman, 2003, Step across this Line. Collected Nonfiction 1992-2002, Modern Library, Nueva York.

Silva Forné, Carlos, Catalina Pérez Correa y Rodrigo Gutiérrez Rivas, 2012, "Uso de la fuerza letal. Muertos, heridos y detenidos en enfrentamientos de las fuerzas federales con presuntos miembros de la delincuencia organizada", en Desacatos. Revista de Antropología Social, núm. 40, pp. 47-64.

Solop, Frederic I. y Nancy A. Wonders, 2012, "The Complexity of Immigration Attitudes in the United States: Public Opinion, Latinos, and the Border States of Arizona and California”, en Mónica Verea (ed.), Sentimientos, acciones y políticas antiinmigrantes. América del Norte y la Unión Europea, Centro de Investigaciones sobre América del Norte-Universidad Nacional Autónoma de México, México, pp. 103-122.

Spener, David, 2009, Clandestine Crossings: Migrants and Coyotes on the Texas-Mexico Border, Cornell University Press, Ithaca.

Stolcke, Verena, 1995, “Talking Culture. New Boundaries, New Rhetorics of Exclusion in Europe”, en Current Anthropology, vol. 36, núm. 1, pp. 1-24. Tuirán, Rodolfo (coord.), 2000, Migración México-Estados Unidos. Presente y futuro, Consejo Nacional de Población, México.

United States Citizenship and Immigration Services (UscIS), 2006, "Secure Fence Act of 2006", en línea: <http://www.uscis.gov/iframe/ilink/ docView/PUBLAW/HTML/PUBLAW/0-0-0-1.html>.

United States Customs and Border Protection (USCBP), 2013a, "Sector Profile-Fiscal Year 2012 (October 1st through September 30th)", United States Customs and Border Protection-Department of Homeland Security, Washington, D. C., en línea: <http://www.cbp.gov/sites/default/files/documents/U.S.\%20Border\%20Patrol\%20Fiscal\%20Year\%202012\%20Sector\%20Profile.pdf>, consultado el 25 de agosto de 2014.

, 2013b, "Border Deaths by Fiscal Year", United States Customs and Border Protection-Department of Homeland Security, Washington, D. C., en línea: <http://www.cbp.gov/sites/default/files/documents/U.S.\%20Border\%20Patro|\%20Fiscal\%20Year\%202012\%20Sector\%20Profile.pdf>, consultado el 25 de agosto de 2014.

, 2013c, "On a Typical Day in Fy 2013, cBP", United States Customs and Border Protection-Department of Homeland Security, Washington, D. C., en línea: <http://www.cbp.gov/newsroom/stats/on-a-typical-day-fy2013>, consultado el 25 de agosto de 2014.

United States Department of Homeland Security (UsDHS), 2011, "Immigration Enforcement Actions: 2010", en Annual Report, Office of Immigration Statistics, en línea: <www.dhs.gov/xlibrary/assets/statistics/publications/enforcement-ar-2010.pdf>, consultado el 16 de marzo de 2012.

Verea, Mónica (ed.), 2012a, Sentimientos, acciones y políticas antiinmigrantes. América del Norte y la Unión Europea, Centro de Investigaciones sobre América del Norte-Universidad Nacional Autónoma de México, México.

, 2012b, "Aproximaciones teóricas para entender las políticas migratorias restrictivas y los sentimientos antiinmigrantes en el siglo xxi", en Mónica Verea (ed.), Sentimientos, acciones y políticas antiinmigrantes. América del Norte y la Unión Europea, Centro de Investigaciones sobre América del Norte-Universidad Nacional Autónoma de México, México, pp. 39-62. 